\title{
Retrieval Analysis of the Emission Spectrum of WASP-12b: Sensitivity of Outcomes to Prior Assumptions and Implications for Formation History
}

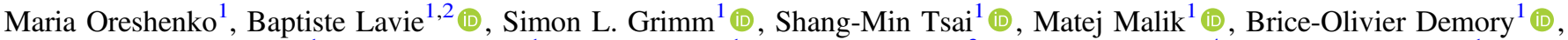 \\ Christoph Mordasini ${ }^{1}$ (D), Yann Alibert ${ }^{1}$ (D), Willy Benz ${ }^{1}$, Sascha P. Quanz ${ }^{3}$ (D), Roberto Trotta ${ }^{4}$ (D), and Kevin Heng ${ }^{1}$ (iD \\ ${ }^{1}$ University of Bern, Center for Space and Habitability, Gesellschaftsstrasse 6, CH-3012 Bern, Switzerland \\ maria.oreshenko@csh.unibe.ch, kevin.heng@csh.unibe.ch \\ ${ }^{2}$ Observatoire de l'Université de Genève, 51 chemin des Mailettes, CH-1290 Sauverny, Switzerland \\ ${ }^{3}$ ETH Zürich, Department of Physics, Wolfgang-Pauli-Strasse 27, CH-8093 Zürich, Switzerland \\ ${ }^{4}$ Astrophysics Group, Imperial College London, Blackett Laboratory, Prince Consort Road, London SW7 2AZ, UK \\ Received 2017 June 15; revised 2017 August 15; accepted 2017 September 1; published 2017 September 14
}

\begin{abstract}
We analyze the emission spectrum of the hot Jupiter WASP-12b using our HELIOS-R retrieval code and HELIOS-K opacity calculator. When interpreting Hubble and Spitzer data, the retrieval outcomes are found to be prior-dominated. When the prior distributions of the molecular abundances are assumed to be log-uniform, the volume mixing ratio of $\mathrm{HCN}$ is found to be implausibly high. A VULCAN chemical kinetics model of WASP-12b suggests that chemical equilibrium is a reasonable assumption even when atmospheric mixing is implausibly rigorous. Guided by (exo)planet formation theory, we set Gaussian priors on the elemental abundances of carbon, oxygen, and nitrogen with the Gaussian peaks being centered on the measured $\mathrm{C} / \mathrm{H}, \mathrm{O} / \mathrm{H}$, and $\mathrm{N} / \mathrm{H}$ values of the star. By enforcing chemical equilibrium, we find substellar $\mathrm{O} / \mathrm{H}$ and stellar to slightly superstellar $\mathrm{C} / \mathrm{H}$ for the dayside atmosphere of WASP-12b. The superstellar carbon-to-oxygen ratio is just above unity, regardless of whether clouds are included in the retrieval analysis, consistent with Madhusudhan et al. Furthermore, whether a temperature inversion exists in the atmosphere depends on one's assumption for the Gaussian width of the priors. Our retrieved posterior distributions are consistent with the formation of WASP-12b in a solar-composition protoplanetary disk, beyond the water iceline, via gravitational instability or pebble accretion (without core erosion) and migration inward to its present orbital location via a disk-free mechanism, and are inconsistent with both in situ formation and core accretion with disk migration, as predicted by Madhusudhan et al. We predict that the interpretation of James Webb Space Telescope WASP-12b data will not be prior-dominated.
\end{abstract}

Key words: planets and satellites: atmospheres

\section{Introduction}

WASP-12b is a well-studied hot Jupiter that has generated ample debate and controversy in the published literature. With an equilibrium temperature in excess of $2500 \mathrm{~K}$ (Hebb et al. 2009), it serves as a high-temperature laboratory for the study of atmospheric chemistry. We expect equilibrium chemistry to be a reasonable approximation, as the high temperatures should overwhelm disequilibrium due to atmospheric circulation or photochemistry. Figure 1 shows a chemical kinetics model of WASP-12b computed using our open-source VULCAN code (Tsai et al. 2017), which lends support to this expectation. Even with an eddy mixing coefficient of $K_{\mathrm{zz}} \sim 10^{12} \mathrm{~cm}^{2} \mathrm{~s}^{-1}$, the model atmosphere is well-described by chemical equilibrium. ${ }^{5}$ Later in the study, we will demonstrate that enforcing chemical equilibrium as a prior assumption circumvents the debate over whether the inferred molecular abundances in WASP-12b are physically and chemically plausible (Madhusudhan 2012; Stevenson et al. 2014; Heng \& Lyons 2016).

An active topic of interest associated with WASP-12b is the inferred carbon-to-oxygen $(\mathrm{C} / \mathrm{O})$ ratio of its atmosphere, starting with the claim of Madhusudhan et al. (2011) and Madhusudhan (2012) that it equals or exceeds unity based on analyzing its emission spectrum. This inference on the $\mathrm{C} / \mathrm{O}$, if

\footnotetext{
Using a sound speed of $c_{s} \sim 1 \mathrm{~km} \mathrm{~s}^{-1}$ and a pressure scale height of $H \sim 100 \mathrm{~km}$ yields $K_{\mathrm{zz}} \sim c_{s} H \sim 10^{12} \mathrm{~cm}^{2} \mathrm{~s}^{-1}$. This may be considered an upper limit as vertical flow velocities are typically subsonic.
}

true, would imply interesting constraints on the formation and/ or evolutionary history of the exoplanet (Öberg et al. 2011; Ali-Dib et al. 2014; Madhusudhan et al. 2014; Mordasini et al. 2016; Öberg \& Bergin 2016; Ali-Dib 2017; Brewer et al. 2017; Espinoza et al. 2017; Madhusudhan et al. 2017), as the C/O of its star has been measured to be $0.48 \pm 0.08$ (Teske et al. 2014). In fact, when compared to a sample of exoplanet-bearing stars, WASP-12 is unremarkably Sun-like (Teske et al. 2014; Brewer \& Fischer 2016). Line et al. (2014) inferred $\mathrm{C} / \mathrm{O}=0.51$ from their retrieval analysis, but their inferred volume mixing ratio for $\mathrm{CO}_{2}$ was nearly 0.06 , a factor of 26 higher than that for $\mathrm{CO}$, which is chemically implausible unless the metallicity is several orders of magnitude above solar (Madhusudhan 2012; Heng \& Lyons 2016). Stevenson et al. (2014) performed a uniform analysis of Hubble and Spitzer secondary-eclipse data, subjected them to a retrieval analysis and found a bimodal distribution for $\mathrm{C} / \mathrm{O}$. Oxygenrich models were ruled out on the basis of chemical implausibility. By contrast, Kreidberg et al. (2015) ruled out a carbon-rich interpretation from analyzing the transmission spectrum of WASP-12b.

These properties of WASP-12b, and the attention it has garnered in the community, compel us to perform our own retrieval analysis of its emission spectrum, which probes the dayside of the exoplanet. Although no new data are being analyzed in the present study, we add value by offering an independent analysis using our own suite of tools (Fortney et al. 2016). Furthermore, we use updated and previously 


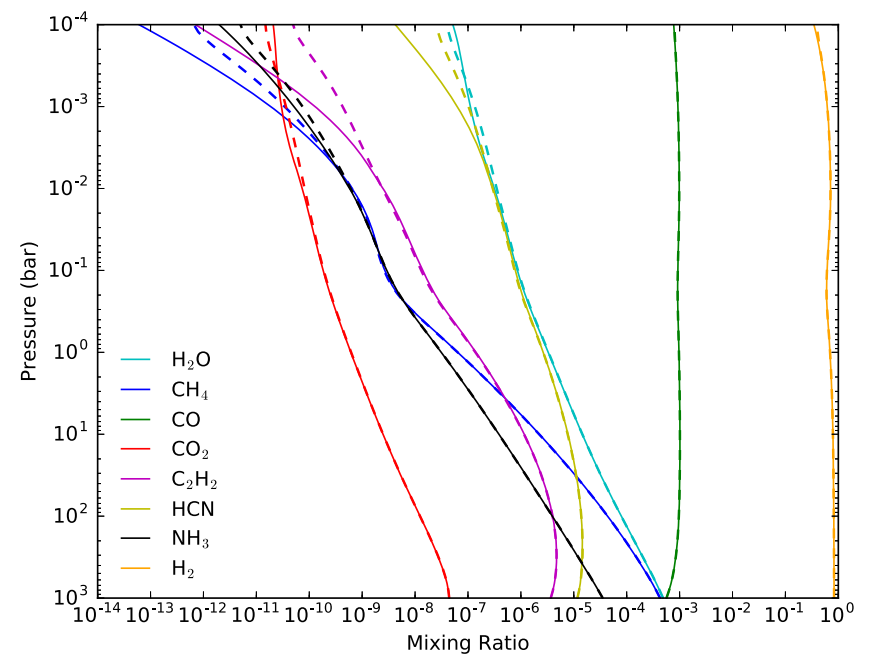

Figure 1. Chemical kinetics model of WASP-12b computed using the opensource VULCAN code (Tsai et al. 2017) and adopting the eddy mixing coefficient to be $K_{\mathrm{zz}}=10^{12} \mathrm{~cm}^{2} \mathrm{~s}^{-1}$ (dashed curves). The solid curves are the molecular abundances in chemical equilibrium. Photochemistry has been omitted, as it is subdominant due to the high temperatures involved. Note that we do not use chemical-equilibrium boundary conditions at the bottom of the model atmosphere, but rather zero-flux boundary conditions. The temperaturepressure profile used is taken from the retrieval model with equilibrium chemistry and no clouds with prior assumptions on the elemental abundances set to twice the measurement errors ("EB, $\times 2$ "; see the text for details). The carbon-to-oxygen ratio is set to unity. Emission spectra typically probe $\sim 0.01-1$ bar, which implies that chemical equilibrium is a good assumption for the atmosphere of WASP- $12 b$.

unavailable and/or unused opacities for $\mathrm{H}_{2} \mathrm{O}$ and $\mathrm{CH}_{4}$. The high-temperature water line lists were published by Barber et al. (2006), while the high-temperature $\mathrm{CH}_{4}$ line lists were published by Yurchenko et al. (2013) and Yurchenko \& Tennyson (2014). For example, Line et al. (2014) did not include $\mathrm{HCN}$ opacities in their retrievals and used nonExoMol $\mathrm{CH}_{4}$ and $\mathrm{H}_{2} \mathrm{O}$ opacities. The studies of Madhusudhan et al. (2011) and Madhusudhan (2012) also did not use the ExoMol $\mathrm{CH}_{4}$ and $\mathrm{H}_{2} \mathrm{O}$ opacities.

In the current study, our focus is on elucidating the dependence of the retrieval outcomes on the prior assumptions set on the metallicity or mixing ratios (relative molecular abundances by number). By "metallicity," we specifically mean the elemental abundances of carbon $(\mathrm{C} / \mathrm{H})$, oxygen $(\mathrm{O} / \mathrm{H})$, and nitrogen $(\mathrm{N} / \mathrm{H})$, since our six-molecule analysis only includes the major carbon-, oxygen-, and nitrogen-bearing species in their gaseous form. The assumptions made on the prior distributions of input parameters is an issue that has not been treated in detail in the literature. Loguniform prior distributions are often assumed (sometimes without explicitly being stated), based on the misconception that they are the most plausible assumption - erroneously termed "uninformative priors" or "uninformed priors"-in the absence of further evidence (Trotta 2008). The key finding of our study is that conclusions, based on analyzing currently available data, drawn on $\mathrm{C} / \mathrm{O}$ and chemistry are strongly tied to our prior assumptions, which are in turn informed by our ideas of physics and chemistry. Given assumptions on the priors, we then interpret the outcomes, using published studies of (exo)planet formation, by assuming that the retrieved elemental abundances are representative of the bulk composition of the exoplanet.

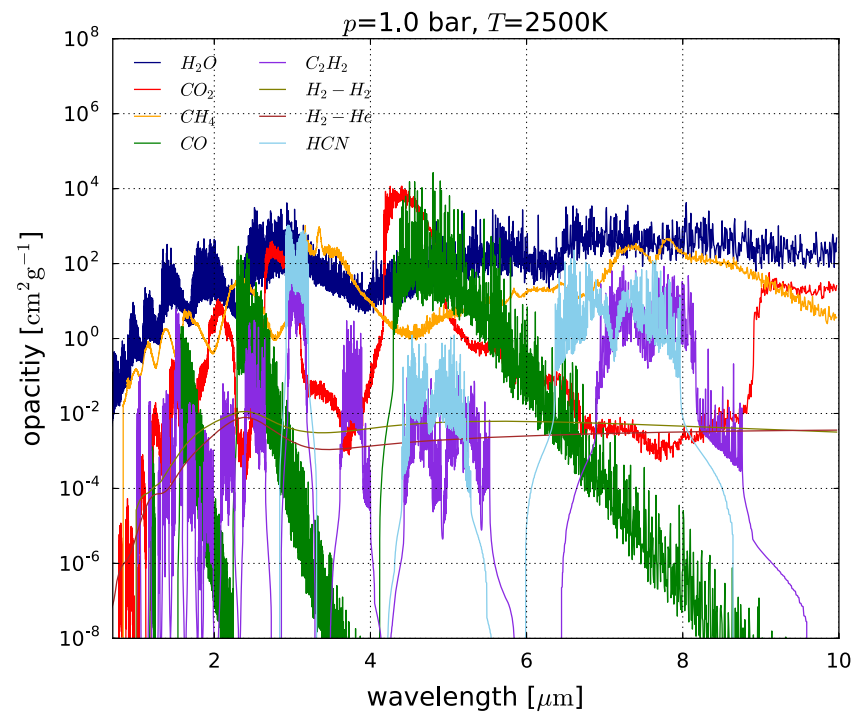

Figure 2. Examples of opacities computed using our HELIOS-K opacity calculator (Grimm \& Heng 2015) for a temperature of $2500 \mathrm{~K}$ and a pressure of 1 bar. The ExoMol database is the source of our $\mathrm{H}_{2} \mathrm{O}$ and $\mathrm{CH}_{4}$ opacities. The $\mathrm{CO}$ and $\mathrm{CO}_{2}$ opacities are from HITEMP, while the $\mathrm{C}_{2} \mathrm{H}_{2}$ and $\mathrm{HCN}$ opacities are from HITRAN.

\section{Methodology}

Our nested-sampling retrieval code, HELIOS-R, and computational setup was previously described in Lavie et al. (2017). The stellar and exoplanetary parameters are taken from Hebb et al. (2009) and Chan et al. (2011). Our nested-sampling (Feroz et al. 2009) retrievals typically use 8 parallel runs of 4000 live points each. The model atmosphere is divided into 100 discrete layers. At every wavelength, the propagation of flux is performed using a direct, analytical solution of the radiative transfer equation in the limit of pure absorption (Heng et al. 2014). The opacities are computed using our customized, open-source opacity calculator, HELIOS-K (Grimm \& Heng 2015), which takes the HITEMP (Rothman et al. 2010), HITRAN (Rothman et al. 1996, 2013), and ExoMol (Barber et al. 2006; Yurchenko et al. 2013; Yurchenko \& Tennyson 2014) spectroscopic databases as inputs to compute the line shapes and strengths. We include the opacities of $\mathrm{CO}, \mathrm{CO}_{2}, \mathrm{CH}_{4}, \mathrm{C}_{2} \mathrm{H}_{2}, \mathrm{H}_{2} \mathrm{O}$, and $\mathrm{HCN}$, as well as collisioninduced absorption associated with $\mathrm{H}_{2}-\mathrm{H}_{2}$ and $\mathrm{H}_{2}-\mathrm{He}$. Figure 2 shows examples of the opacities computed. We use the opacity sampling method with a spectral resolution of $1 \mathrm{~cm}^{-1}$. Our linewing cutoff is $100 \mathrm{~cm}^{-1}$ applied to all of the spectral lines. We use the analytical temperature-pressure profiles originally derived by Guillot (2010), and later generalized to include scattering by Heng et al. (2012) and Heng et al. (2014). These profiles enforce radiative equilibrium (local energy conservation) by construction, but are too isothermal at high altitudes due to the assumption that the Planck, absorption, and flux mean opacities are equal. By numerical experimentation (not shown), we find that the temperature-pressure profile in the limit of pure absorption suffices for our purposes, which is to describe the shape of the profile with as few parameters as possible: $\kappa_{\mathrm{IR}}$ (the mean infrared opacity associated with the temperature-pressure profile) and $\gamma$ (the "greenhouse parameter," which is the ratio of the mean optical/visible to mean infrared opacities). There is no attempt to seek self-consistency between these parameters and the 
wavelength-dependent opacities used. ${ }^{6}$ Atmospheres without and with temperature inversions have $\gamma<1$ and $\gamma>1$, respectively.

For chemistry, we consider two types of models: unconstrained and equilibrium chemistry. The former is the typical approach, which assumes mixing ratios that are constant throughout the atmosphere and uses them as fitting parameters. In other words, no chemistry is actually being considered. The latter enforces chemical equilibrium via the analytical formulae of Heng \& Tsai (2016), who validated these formulae against calculations of Gibbs free energy minimization and demonstrated that they are accurate at the $\sim 1 \%$ level or better. For chemical-equilibrium models, the fitting parameters are $\mathrm{C} / \mathrm{H}$, $\mathrm{O} / \mathrm{H}$, and $\mathrm{N} / \mathrm{H}$. The prior distribution of $\mathrm{C} / \mathrm{O}$ is roughly uniform, unlike for unconstrained chemistry where it is doublepeaked (Line et al. 2013). In chemical equilibrium, specifying the elemental abundances allows all of the molecular abundances to be computed, with no parametric freedom, given a temperature and pressure.

We are agnostic about the terms "cloud" and "haze" and use them interchangeably for this study. ${ }^{7}$ We implement the simplified cloud model introduced by Lee et al. (2013) and used by Lavie et al. (2017), which describes a monodisperse population of spherical cloud particles with radii $r_{\mathrm{c}}$, cloud volume mixing ratio $f_{\text {cloud }}$ and a single composition (represented by the parameter $Q_{0}$ ). Refractory and volatile cloud species have $Q_{0} \sim 1$ and $\sim 10$, respectively. This cloud model accommodates both small and large particles, and correctly reproduces the limits of Rayleigh and gray scattering. It is based on the notion that curves of the extinction coefficient have a roughly universal shape (Pierrehumbert 2010).

\section{Results}

We begin by presenting a pair of retrieval models that make the common assumption of log-uniform priors (Figure 3). For the measured emission spectrum of WASP-12b, we use the published data of Stevenson et al. (2014) as stated in their Table 3. For the model with unconstrained chemistry, we set log-uniform priors on the six mixing ratios. For the model with equilibrium chemistry, we set log-uniform priors on the elemental abundances of carbon, oxygen, and nitrogen. As an improvement over the work of Stevenson et al. (2014), we include clouds in our analysis as part of the retrieval (i.e., the cloud parameters are not fixed to preset values). The other parameters also have log-uniform priors, except for $Q_{0}$, which has a (linearly) uniform prior.

In Figure 3, the first thing to notice is that the cloud parameters display degeneracies that match our physical intuition: the mixing ratios are degenerate with cloud composition, particle radius, and number density. In particular, it is possible to set bounds on the cloud particle radius, but the cloud composition is essentially - and unsurprisingly-unconstrained. When we include only $\mathrm{CO}, \mathrm{CO}_{2}, \mathrm{CH}_{4}$, and $\mathrm{H}_{2} \mathrm{O}$ in the retrieval, we reproduce the result of Line et al. (2014) and Stevenson et al. (2014) that unrealistically high abundances for $\mathrm{CO}_{2}$ are obtained (not shown). (Heng \& Lyons 2016 have previously elucidated this implausibility using validated analytical formulae.) Such high abundances of $\mathrm{CO}_{2}$ drive the

\footnotetext{
6 None of the practitioners of atmospheric retrieval are currently able to do this.

7 These terms are either used to distinguish between size (Earth science convention) or formation origin (planetary science convention), and there is no consensus within the exoplanet community on their usage.
}

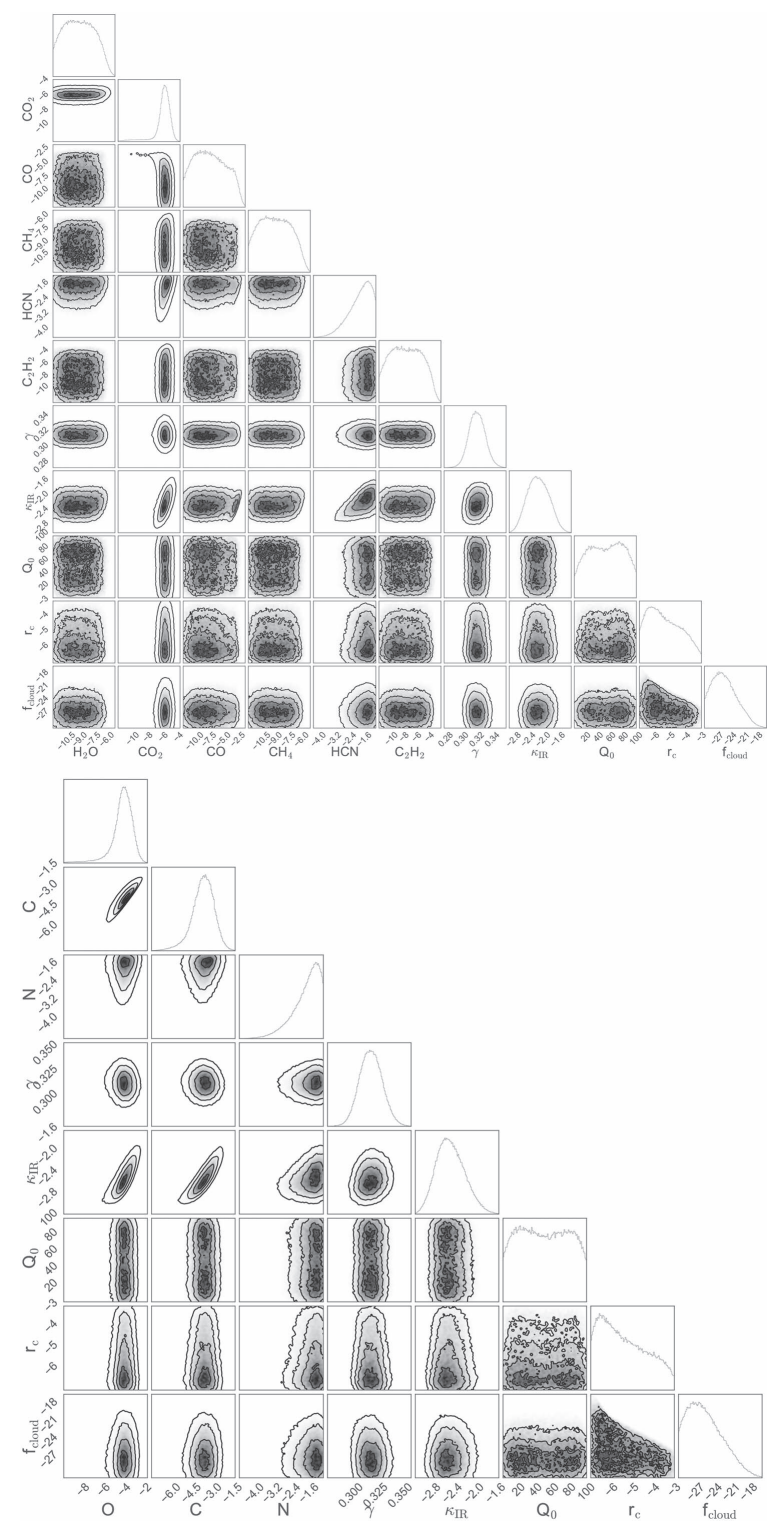

Figure 3. Posterior distributions of fitting parameters for cloudy retrieval models with unconstrained chemistry (top panel) and equilibrium chemistry (bottom panel) with log-uniform priors. $\kappa_{\mathrm{IR}}$ has physical units of $\mathrm{m}^{2} \mathrm{~kg}^{-1}$, while $r_{\mathrm{c}}$ is given in $\mathrm{m}$. The rest of the parameters are dimensionless.

retrieval predominantly toward a solution with $\mathrm{C} / \mathrm{O} \approx 0.5$. Furthermore, the prior distribution of $\mathrm{C} / \mathrm{O}$ is double-peaked at 0.5 and 1 (Line et al. 2013), which appears in the posterior distribution as well (Figure 4).

When $\mathrm{C}_{2} \mathrm{H}_{2}$ and $\mathrm{HCN}$ are included, we obtain the mixing ratio of $\mathrm{HCN}$ to be $\sim 10^{-2}-10^{-1}$ (top panel of Figure 3 ). This is chemically implausible, as suggested by the detailed chemical kinetics calculations of Moses et al. (2013), who estimated an upper limit to the mixing ratio of $\mathrm{HCN}$ of $\sim 10^{-3}$ for $\mathrm{C} / \mathrm{O}<2$ and thrice the solar metallicity. When chemical equilibrium is enforced with log-uniform priors, we obtain $\mathrm{N} / \mathrm{H} \sim 10^{-2}-10^{-1}$ (bottom panel of Figure 3), which is similarly implausible. These anomalies arise because the opacity of $\mathrm{HCN}$ is driving the fit at the wavelengths of the Spitzer photometry (Stevenson et al. 2014; Figure 2). The lesson learned is that the "simplest" assumption made on the prior distributions of fitting parameters may not be the best one (Trotta 2008). Rather, we need to be guided by physics and chemistry. 

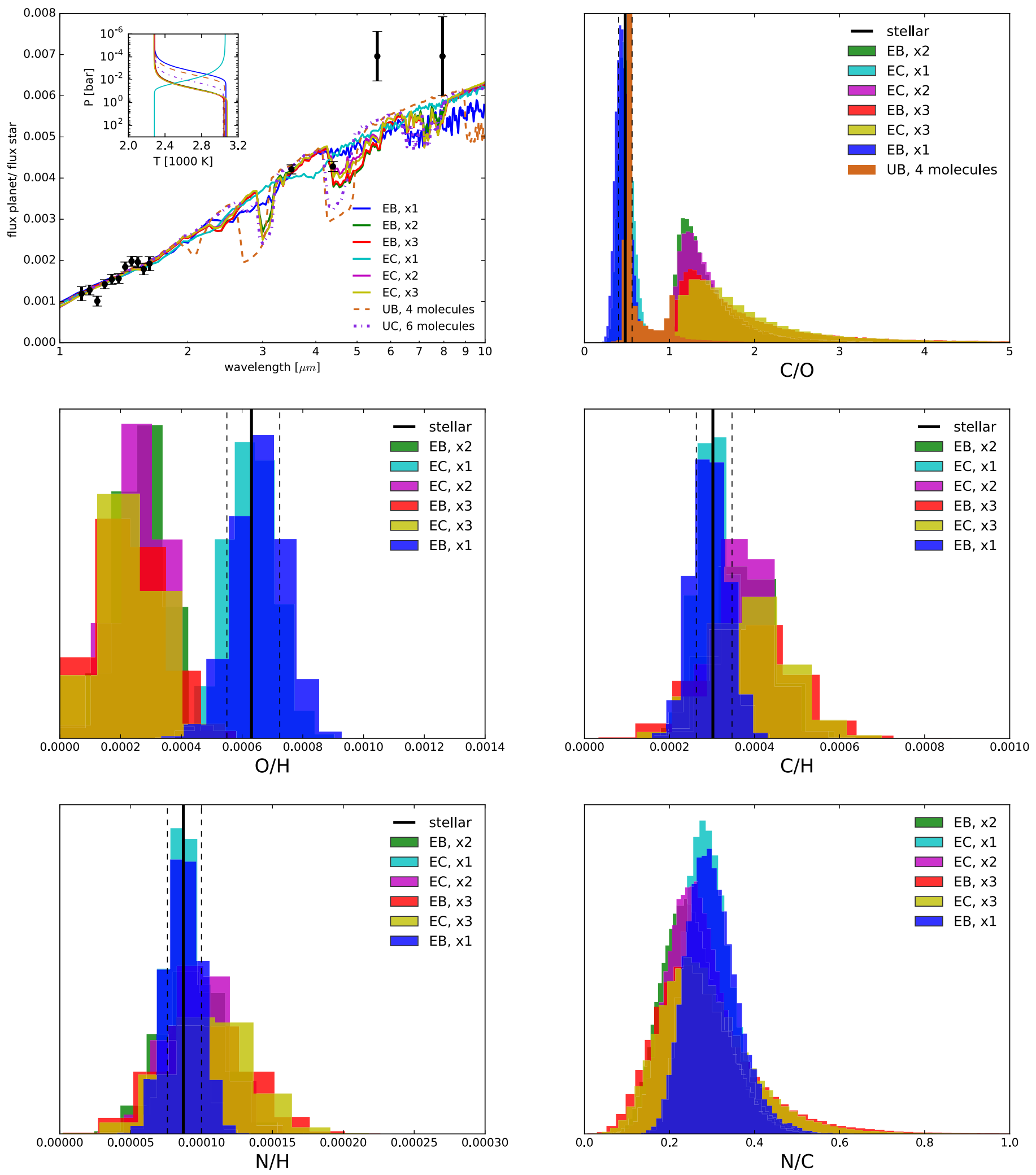

Figure 4. Best-fit spectra (top left panel) and the posterior distributions of $\mathrm{C} / \mathrm{O}$ (top right panel), $\mathrm{O} / \mathrm{H}$ (middle left panel), $\mathrm{C} / \mathrm{H}$ (middle right panel), $\mathrm{N} / \mathrm{H}$ (bottom left panel), and N/C (bottom right panel) for the six retrieval models with equilibrium chemistry. The cloudfree and cloudy models are labeled "EB" and "EC," respectively. The models labeled " $\times 1$, , " $\times 2$," and " $\times 3$ " adopt Gaussian widths on the prior distributions of the elemental abundances that are once, twice, and thrice the measurement errors of the stellar elemental abundances, respectively. The model labeled "UB, 4 molecules" assumes unconstrained chemistry and a cloudfree atmosphere with $\mathrm{CO}, \mathrm{CO}_{2}, \mathrm{H}_{2} \mathrm{O}$, and $\mathrm{CH}_{4}$ only and is included as a reference to models previously published in the literature. The model labeled "UC, 6 molecules" assumes unconstrained chemistry and a cloudy atmosphere and is included for completeness as it gives an unrealistic/unphysical abundance for HCN. The marginal posterior distributions are all normalized to have unity area. Note that the $\mathrm{C} / \mathrm{O} \approx 0.5$ peak for the "UB, 4 molecules" model extends beyond the plot and we have truncated it for clarity. 
Motivated by the calculations in Figure 1, we enforce chemical equilibrium as a prior. Instead of log-uniform priors, we now set Gaussian priors on the elemental abundances, based on the measured ${ }^{8}$ WASP-12 values by Teske et al. (2014): $\mathrm{C} / \mathrm{H}_{\star}=3.02_{-0.39}^{+0.45} \times 10^{-4}$ and $\mathrm{O} / \mathrm{H}_{\star}=6.31_{-0.81}^{+0.93} \times 10^{-4}$. Since Teske et al. (2014) did not report measured $\mathrm{N} / \mathrm{H}$ values, we use $\mathrm{N} / \mathrm{O}_{\odot}=0.138$ (Lodders 2003) to transform $\mathrm{O} / \mathrm{H}$ into $\mathrm{N} / \mathrm{H}_{\star}=8.71_{-1.12}^{+1.28} \times 10^{-5}$. We additionally compute models with Gaussian widths that are twice and thrice the measurement errors. The top panel of Figure 4 shows that the cloudfree model with $\times 1$ the measurement error as the Gaussian width produces a posterior distribution of $\mathrm{C} / \mathrm{O}$ that is unsurprisingly peaked at the measured $\mathrm{C} / \mathrm{O}_{\star}=0.48$ value of WASP-12. In other words, we simply reproduce the (tight) prior. Of greater interest are the posterior distributions when the widths of the Gaussian priors are doubled or tripled, which peak just above a $\mathrm{C} / \mathrm{O}$ value of unity and trail off as it becomes $2-3$. This outcome of a carbon-rich dayside atmosphere of WASP-12b is independent of whether clouds are included in the analysis, because the cloud layer is optically thin. The posterior distribution of $\mathrm{O} / \mathrm{H}$ is substellar, while that of $\mathrm{C} / \mathrm{H}$ is slightly superstellar but still consistent with being stellar. Our posterior distributions for $\mathrm{C} / \mathrm{H}, \mathrm{O} / \mathrm{H}$, and $\mathrm{C} / \mathrm{O}$ are broadly consistent with those reported by Madhusudhan et al. (2011, 2014). We note that increasing the Gaussian widths of the priors to eight times the measurement errors does not alter our qualitative conclusions (not shown).

Another surprising outcome of this set of six retrievals is the shape of the temperature-pressure profile (bottom panel of Figure 4). While the best-fit spectra look similar among the six different cases, the temperature-pressure profile for the cloudy $\times 1$ model exhibits a temperature inversion that is entirely driven by the retrieval attempting to fit the four Spitzer photometric points. When the Gaussian width on the priors is doubled or tripled, the temperature inversion disappears. For illustration, the top panel of Figure 5 shows the posterior distributions for the cloudy case with $\times 2$ the measurement errors for the Gaussian width of the priors.

\section{Discussion \\ 4.1. Implications for Formation and Comparison to Previous Studies}

Generally, it is challenging to make a hot Jupiter with substellar O/H (Brewer et al. 2017). Several studies have previously explored the link between the formation and migration history of hot Jupiters and their atmospheric chemistry. Madhusudhan et al. (2014) predicted that the formation of gas-giant exoplanets at large orbital distances via gravitational instability, from a solar-composition protoplanetary disk, and their subsequent migration inward via diskfree mechanisms produces hot Jupiters with stellar $\mathrm{C} / \mathrm{H}$, substellar $\mathrm{O} / \mathrm{H}$, and superstellar $\mathrm{C} / \mathrm{O}$. Our retrieval outcomes are consistent with this scenario. If the disk is instead constructed with molecular abundances based on observations of ice and gas in protoplanetary disks (Öberg et al. 2011), then it produces hot Jupiters with $\mathrm{C} / \mathrm{H}$ and $\mathrm{O} / \mathrm{H}$ that are both substellar. Core accretion with disk-free migration produces $\mathrm{C} / \mathrm{H}$ and $\mathrm{O} / \mathrm{H}$ that are either both substellar, both stellar, or both superstellar-neither of these scenarios are consistent with

\footnotetext{
8 Since these priors are based on measurements, they could alternatively be considered as being part of the likelihood.
}
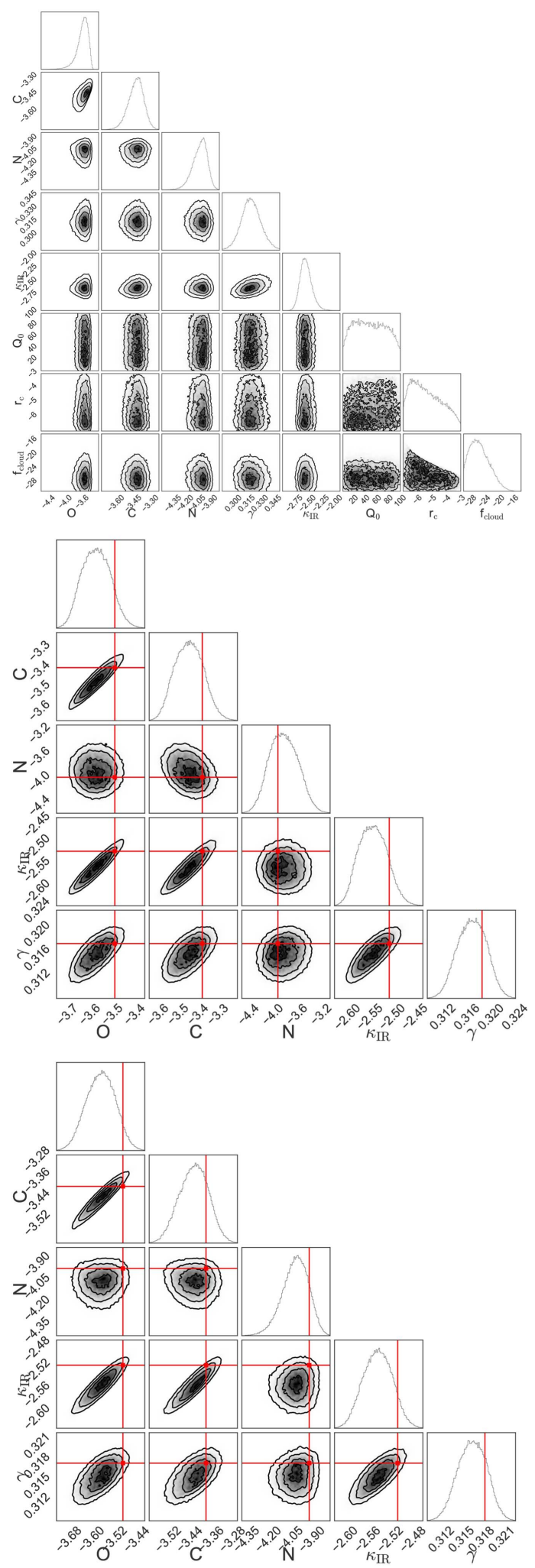

Figure 5. Posterior distributions of fitting parameters for the equilibriumchemistry model. $\kappa_{\mathrm{IR}}$ has physical units of $\mathrm{m}^{2} \mathrm{~kg}^{-1}$, while $r_{\mathrm{c}}$ is given in $\mathrm{m}$. The rest of the parameters are dimensionless. Top panel: retrieval on WFC3 and Spitzer data with a Gaussian width on the prior distribution of the elemental abundances that is twice the measurement error of the stellar elemental abundances (i.e., the "EC, $\times 2$ " model). Middle panel: retrieval on mock JWST data (see the text for more details) for $\mathrm{EB}, \times 2$ cloudfree model. Bottom panel: cloudfree retrieval on mock JWST data with log-uniform priors. For the retrievals on mock data, the input values of parameters are given by the straight lines. 
our retrieval outcomes. Core accretion with disk migration produces superstellar values for both $\mathrm{C} / \mathrm{H}$ and $\mathrm{O} / \mathrm{H}$.

An active topic of debate concerns the role of pebbles in the protoplanetary disk (Ormel \& Klahr 2010; Lambrechts \& Johansen 2012). Pebbles are intermediate-sized solids with Stokes numbers on the order of unity, which are imperfectly coupled to the disk gas; their exact sizes are a function of the local conditions of the disk. The drift of pebbles across the $\mathrm{CO}_{2}, \mathrm{CO}$, and $\mathrm{H}_{2} \mathrm{O}$ snowlines is capable of locally altering the values of $\mathrm{C} / \mathrm{H}, \mathrm{O} / \mathrm{H}$, and $\mathrm{C} / \mathrm{O}$ in a disk (Öberg \& Bergin 2016). The key difference between pebbles and regular planetesimals is that, to zeroth order, pebbles are purportedly able to accrete onto the core of the exoplanet directly without polluting the atmosphere, implying that the elemental abundances range from being substellar to stellar. In the scenario depicted by Madhusudhan et al. (2017), hot Jupiters accrete most of their gas within the $\mathrm{H}_{2} \mathrm{O}$ snowline (Ali-Dib et al. 2014), which naturally yields a stellar $\mathrm{C} / \mathrm{H}$, substellar $\mathrm{O} / \mathrm{H}$, and superstellar $\mathrm{C} / \mathrm{O} \approx 0.7-0.8$. At face value, this is at odds with our finding that $\mathrm{C} / \mathrm{O} \approx 1-2$. Any erosion of the core tends to drive $\mathrm{C} / \mathrm{H}$ and $\mathrm{O} / \mathrm{H}$ to superstellar values and $\mathrm{C} / \mathrm{O}$ to substellar values, further increasing the discrepancy between the theoretical prediction and our inferred posterior distributions. An alternative scenario is that WASP-12b formed at large orbital distances (as a cold Jupiter) via pebble accretion and migrated inward via a disk-free mechanism. In such a scenario, Madhusudhan et al. (2017) predict $\mathrm{O} / \mathrm{H} \approx 0.2-0.5 \mathrm{O} / \mathrm{H}_{\star}$, $\mathrm{C} / \mathrm{H} \approx 0.5-0.9 \mathrm{C} / \mathrm{H}_{\star}$, and $\mathrm{C} / \mathrm{O} \approx 1$. Our retrieved posterior distributions are consistent with such a scenario. Based on the inferred substellar $\mathrm{O} / \mathrm{H}$ and superstellar $\mathrm{C} / \mathrm{O}$ values, Brewer et al. (2017) claimed another hot Jupiter, HD 209458b, to also have undergone disk-free migration.

We note that WASP-12b is part of a triple-star system (Bechter et al. 2014) and has a measured spin-orbit alignment of $59_{-20}^{+15}$ degrees, which may be consistent with the disk-free migration scenario.

Our retrieved posterior distributions are inconsistent with the in situ formation of WASP-12b (Batygin et al. 2016; Boley et al. 2016), which Madhusudhan et al. (2017) predict to yield $\mathrm{O} / \mathrm{H} \approx 0.8-1.5 \mathrm{O} / \mathrm{H}_{\star}, \mathrm{C} / \mathrm{H} \approx \mathrm{C} / \mathrm{H}_{\star}$, and $\mathrm{C} / \mathrm{O} \approx 0.4-0.7$. Ali-Dib (2017) suggests that to produce $\mathrm{C} / \mathrm{O} \gtrsim 1$ via in situ formation requires that the parent star has $\mathrm{C} / \mathrm{O} \approx 0.8$.

Generally, our finding of substellar values for $\mathrm{O} / \mathrm{H}$ provides counter-evidence against late-time planetesimal accretion or core erosion. Both processes would enrich the atmosphere of WASP-12b to beyond its stellar values. Furthermore, the posterior distribution of $\mathrm{N} / \mathrm{C}$, which is consistent with being solar (Lodders 2003), provides clues on the original site of formation in the outer protoplanetary disk (Öberg \& Bergin 2016).

\subsection{Are Retrievals of James Webb Space Telescope (JWST) Data in the Prior-dominated Regime?}

Our findings beg the question: are retrievals of JWST spectra also in the prior-dominated regime? To address it specifically for WASP-12b, we produce mock spectra with a resolution of 100 over the wavelength range of $0.7-5 \mu \mathrm{m}$. We assume measurement uncertainties of $100 \mathrm{ppm}$. The middle and bottom panels of Figure 5 show the posterior distributions of parameters from retrievals assuming log-uniform and Gaussian priors, respectively. In both cases, the retrieved parameter values are essentially the same and within $\sim 30 \%$ of the true (input) values, suggesting that the interpretation of JWST spectra will not be in the prior-dominated regime.

We acknowledge partial financial support from the Center for Space and Habitability (CSH), the PlanetS National Center of Competence in Research (NCCR), the Swiss National Science Foundation, and the Swiss-based MERAC Foundation.

\section{ORCID iDs}

Baptiste Lavie (iD https://orcid.org/0000-0001-8884-9276 Simon L. Grimm (iD https://orcid.org/0000-0002-0632-4407 Shang-Min Tsai (iD https://orcid.org/0000-0002-8163-4608 Matej Malik (iD https://orcid.org/0000-0002-2110-6694 Brice-Olivier Demory (i) https://orcid.org/0000-00029355-5165

Christoph Mordasini (1D https://orcid.org/0000-00021013-2811

Yann Alibert (1D https://orcid.org/0000-0002-4644-8818

Sascha P. Quanz (10 https://orcid.org/0000-0003-3829-7412

Roberto Trotta (iD https://orcid.org/0000-0002-3415-0707

Kevin Heng (ib https://orcid.org/0000-0003-1907-5910

\section{References}

Ali-Dib, M. 2017, MNRAS, 467, 2845

Ali-Dib, M., Mousis, O., Petit, J.-M., \& Lunine, J. I. 2014, ApJ, 785, 125

Barber, R. J., Tennyson, J., Harris, G. J., \& Tolchenov, R. N. 2006, MNRAS, 368, 1087

Batygin, K., Bodenheimer, P. H., \& Laughlin, G. P. 2016, ApJ, 829, 114

Bechter, E. B., Crepp, J. R., Ngo, H., et al. 2014, ApJ, 788, 2

Boley, A. C., Granados Contreras, A. P., \& Gladman, B. 2016, ApJL, 817, L17

Brewer, J. M., \& Fischer, D. A. 2016, ApJ, 831, 20

Brewer, J. M., Fischer, D. A., \& Madhusudhan, N. 2017, AJ, 153, 83

Chan, T., Ingemyr, M., Winn, J. N., et al. 2011, AJ, 141, 179

Espinoza, N., Fortney, J. J., Miguel, Y., Thorngren, D., \& Murray-Clay, R. 2017, ApJL, 838, L9

Feroz, F., Hobson, M. P., \& Bridges, M. 2009, MNRAS, 398, 1601

Fortney, J. J., Robinson, T. D., Domagal-Goldman, S., et al. 2016, arXiv:1602. 06305

Grimm, S. L., \& Heng, K. 2015, ApJ, 808, 182

Guillot, T. 2010, A\&A, 520, A27

Hebb, L., Collier-Cameron, A., Loeillet, B., et al. 2009, ApJ, 693, 1920

Heng, K., Hayek, W., Pont, F., \& Sing, D. K. 2012, MNRAS, 420, 20

Heng, K., \& Lyons, J. R. 2016, ApJ, 817, 149

Heng, K., Mendonça, J. M., \& Lee, J.-M. 2014, ApJS, 215, 4

Heng, K., \& Tsai, S.-M. 2016, ApJ, 829, 104

Kreidberg, L., Line, M. R., Bean, J. L., et al. 2015, ApJ, 814, 66

Lambrechts, M., \& Johansen, A. 2012, A\&A, 544, A32

Lavie, B., Mendonça, J. M., Mordasini, C., et al. 2017, AJ, 154, 91

Lee, J.-M., Heng, K., \& Irwin, P. G. J. 2013, ApJ, 778, 97

Line, M. R., Knutson, H., Wolf, A. S., \& Yung, Y. L. 2014, ApJ, 783, 70

Line, M. R., Wolf, A. S., Zhang, X., et al. 2013, ApJ, 775, 137

Lodders, K. 2003, ApJ, 591, 1220

Madhusudhan, N. 2012, ApJ, 758, 36

Madhusudhan, N., Amin, M. A., \& Kennedy, G. M. 2014, ApJL, 794, L12

Madhusudhan, N., Betram, B., Johansen, A., \& Eriksson, L. 2017, MNRAS, 469, 4102

Madhusudhan, N., Crouzet, N., McCullough, P. R., Deming, D., \& Hedges, C. 2014, ApJL, 791, L9

Madhusudhan, N., Harrington, J., Stevenson, K. B., et al. 2011, Natur, 469, 64

Mordasini, C., van Boekel, R., Mollière, P., Henning, Th., \& Benneke, B. 2016, ApJ, 832, 41

Moses, J. I., Madhusudhan, N., Visscher, C., \& Freedman, R. S. 2013, ApJ, 763,25

Öberg, K. I., \& Bergin, E. A. 2016, ApJL, 831, L19

Öberg, K. I., Murray-Clay, R., \& Bergin, E. A. 2011, ApJL, 743, L16

Ormel, C. W., \& Klahr, H. H. 2010, A\&A, 520, A43 
Pierrehumbert, R. T. 2010, Principles of Planetary Climate (New York: Cambridge Univ. Press)

Rothman, L. S., Gordon, I. E., Babikov, Y., et al. 2013, JQSRT, 130, 4

Rothman, L. S., Gordon, I. E., Barber, R. J., et al. 2010, JQSRT, 111, 2139

Rothman, L. S., Rinsland, C. P., Goldman, A., et al. 1996, JQSRT, 60, 665

Stevenson, K. B., Bean, J. L., Madhusudhan, N., \& Harrington, J. 2014, ApJ, 791,36
Teske, J. K., Cunha, K., Smith, V. V., Schuler, S. C., \& Griffith, C. A. 2014, ApJ, 788, 39

Trotta, R. 2008, ConPh, 49, 71

Tsai, S.-M., Lyons, J. R., Grosheintz, L., Rimmer, P. B., Kitzmann, D., \& Heng, K. 2017, ApJS, 228, 20

Yurchenko, S. N., \& Tennyson, J. 2014, MNRAS, 440, 1649

Yurchenko, S. N., Tennyson, J., Barber, R. J., \& Thiel, W. 2013, JMoSp, 291, 69 